\title{
SOME COUNTEREXAMPLES IN THE CLASSIFICATION OF OPEN RIEMANN SURFACES ${ }^{1}$
}

\author{
H. L. ROYDEN
}

Recently Ahlfors and the author [1] constructed a Riemann surface of hyperbolic type which possessed no nonconstant harmonic functions with a finite Dirichlet integral. In the first section we explore some of the consequences of this example and construct a Riemann surface on which the spaces $H D$ and $H B D$ have dimension $n$. In the next section a bounded Riemann surface is exhibited which has no $H D$ functions on it which vanish on the relative boundary, while it has a nonconstant $H D$ whose normal derivative vanishes on the relative boundary. In the last section we use a refinement of the method in [1] to construct a Riemann surface admitting a nonconstant bounded harmonic function, but no nonconstant harmonic functions with a finite Dirichlet integral, thus demonstrating that the classes $O_{H B}$ and $O_{H D}$ are distinct.

1. Consider a sequence $1 / 2<r_{1}<\cdots<r_{n}<\cdots<1$ and the segments

$$
\begin{aligned}
& \Delta_{n}^{h}: \quad\left\{\begin{array}{l}
r_{n} \leqq r \leqq r_{n+1}, \\
\theta=2 \pi h \cdot 2^{-n}
\end{array}\right. \\
& 0 \leqq h<2^{n} .
\end{aligned}
$$

We divide each $\Delta_{n}^{n}$ into $2^{n}$ subsegments $\Delta_{n}^{n, k}$ of equal logarithmic length and form a Riemann surface $W$ by identifying the left edge of $\Delta_{n}^{h, \boldsymbol{k}}$ with the right edge of $\Delta_{n}^{h+\boldsymbol{k}, \boldsymbol{k}}$, where it is to be understood that $h+k$ is reduced to its remainder $\bmod 2^{n}$. It was. shown in [1] that $W$ has no nonconstant harmonic functions with a finite Dirichlet integral defined on it, provided

$$
\sup 2^{n} \log \frac{1}{r_{n}}=\infty .
$$

Let $V$ be the surface formed by removing the circle $K: r<1 / 2$ from $W$, and denote the circumference $r=1 / 2$ by $R$. We use $H N$ $=H N(V)$ to denote the space of harmonic functions on $V$ which have a finite Dirichlet integral and whose normal derivative vanishes on $R$ and use $H O=H O(V)$ to denote the space of those functions which have a finite Dirichlet integral and which vanish on $R$.

Presented to the Society, September 5, 1952; received by the editors July 18, 1952. Task V.

1 Work sponsored by the Office of Naval Research under contract N6ori-106 
By Theorem 10 of [2] the space $H B D(W)$ of those harmonic functions on $W$ which have a finite Dirichlet integral and are bounded is isomorphic to

$$
\Psi O(V)+H O(K)
$$

and to

$$
H N(V)+H N(K) \text {. }
$$

Since $W$ has a Green's function, we include the constants in $H B D$ by convention. Hence $H B D$ consists of the constants alone and is thus one-dimensional. The compactness of $K$ implies that $H O(K)$ and $H N(K)$ contain only the function zero. Remembering that $H B D$ is dense in $H D$ in the sense of the Dirichlet metric [2], we also have $H D$ one-dimensional and hence:

The spaces $H O(V)$ and $H N(V)$ have dimension one. Consequently $H O(V)$ consists of multiples of $\log (r / 2)$ while $H N(V)$ consists of constants.

If we reflect $V$ in the circle $R$, we obtain a surface $W_{2}$ on which the space $H D$ has dimension 2. Thus if $u$ is harmonic and has a finite Dirichlet integral we must have

$$
u=C_{1}+C_{2} \log r .
$$

Similarly, if we start from the complex sphere from which $n+1$ circular disks have been removed and attach a replica of $V$ in place of each disk, we obtain a surface $W_{n}$ with the property that $H B D\left(W_{n}\right)$ is $n$-dimensional. Thus:

For each integer $n$ there exists a Riemann surface $W_{n}$ on which the spaces $H D\left(W_{n}\right)$ and $H B D\left(W_{n}\right)$ are n-dimensional.

2. Consider a sequence $0<\eta_{1}<\cdots<\eta_{n}<\cdots<1$, set $\eta_{-n}=-\eta_{n}$, and form the segments

$$
\Delta_{n}^{h}:\left\{\begin{array}{l}
\eta_{n} \leqq y \leqq \eta_{n+1}, \\
x=(h+1) 2^{-|n|}, \quad 0 \leqq h \leqq 2^{|n|}-1 .
\end{array}\right.
$$

We divide each $\Delta_{n}^{n}$ into $2^{n}$ subsegments $\Delta_{n}^{n, k}$ of equal lengths and construct a bounded Riemann surface $V^{\prime}$ from the interior of the rectangle $|y| \leqq 1,0 \leqq x \leqq 1$, by identifying the left edge of $\Delta_{n}^{h, k}$ with the right edge of $\Delta_{n}^{n+k, k}$, where here it is understood that $h+k$ is reduced to its remainder mod $2^{|n|}-1$. The relative boundary $R$ of $V^{\prime}$ consists of the segments $x=0$ and $x=1$.

Let $u$ be a harmonic function defined on $V^{\prime}$ which has a finite Dirichlet integral and vanishes on $R$. Then, given $\delta>0$, there is a set 
$E_{8}$ of measure greater than $1-\delta$ such that

$$
\int_{-1}^{1}\left[\left(\frac{\partial u}{\partial x}\right)^{2}+\left(\frac{\partial u}{\partial y}\right)^{2}\right] d y<M_{3}
$$

for $x \in E_{\delta}$. Now

$$
\left|u\left(x, y_{2}\right)-u\left(x, y_{1}\right)\right|^{2}=\left(\int_{y_{1}}^{y_{2}} \frac{\partial u}{\partial y} d y\right)^{2} \leqq\left(y_{2}-y_{1}\right) M_{8}
$$

by the Schwarz inequality, and hence there is a function $l(x)$ such that

$$
\lim _{y \rightarrow 1} u(x, y)=l(x)
$$

uniformly for $x \in E_{\delta}$. Thus for $\epsilon>0$, we may choose $N$ so that $l(x)^{2}$ $\leqq u(x, y)^{2}+\epsilon$ for all $x \in E_{\delta}$ and all $y \geqq \eta_{N}$.

Since the right edge of the interval $\Delta_{n}^{h, n}$ is identified with the left edge of $\Delta_{n}^{0, n}$, we have

$$
u(x, y)=\int_{0}^{2^{-n}} \frac{\partial u}{\partial x} d x+\int_{(h+1) 2^{-n}}^{x} \frac{\partial u}{\partial x} d x
$$

for $(h+1) 2^{-n} \leqq x \leqq(h+2) 2^{-n}$ and for $y$ in the projection of $\Delta_{n}^{0, h}$. By the Schwarz inequality

$$
u(x, y)^{2} \leqq 2^{-n} \int_{0}^{2^{-n}}\left(\frac{\partial u}{\partial x}\right)^{2} d x+2^{-n} \int_{(n+1) 2-\infty}^{x}\left(\frac{\partial u}{\partial x}\right)^{2} d x
$$

whence

$$
u^{2} \leqq 2^{-n+1} \int_{0}^{1}\left[\left(\frac{\partial u}{\partial x}\right)^{2}+\left(\frac{\partial u}{\partial y}\right)^{2}\right] d x .
$$

For $n \geqq N$ and $x$ in $I_{n}$, the intersection of $E_{8}$ with the interval

$$
(h+1) 2^{-n} \leqq x \leqq(h+2) 2^{-n},
$$

we have also

$$
l(x)^{2} \leqq 2^{-n+1} \int_{0}^{1}\left[\left(\frac{\partial u}{\partial x}\right)^{2}+\left(\frac{\partial u}{\partial y}\right)^{2}\right] d x+\epsilon .
$$

Integrating with respect to $y$ in the projection $P^{h}$ of $\Delta_{a}^{0, \lambda}$ gives

$$
\begin{aligned}
2^{-n}\left(\eta_{n+1}-\eta_{n}\right) l(x)^{2} \leqq & 2^{-n+1} \int_{P^{h}} \int_{0}^{1}\left[\left(\frac{\partial u}{\partial x}\right)^{2}+\left(\frac{\partial u}{\partial y}\right)^{2}\right] d x d y \\
& +2^{-n}\left(\eta_{n+1}-\eta_{n}\right) .
\end{aligned}
$$


Integrating with respect to $h$ in $I_{n}$ gives

$$
\begin{aligned}
& 2^{-n}\left(\eta_{n+1}-\eta_{n}\right) \int_{I_{n}} l(x)^{2} d x \leqq 2^{-2 n+1} \int_{P^{n}} \int_{0}^{1}\left[\left(\frac{\partial u}{\partial x}\right)^{2}+\left(\frac{\partial u}{\partial y}\right)^{2}\right] d x d y \\
& +\epsilon 2^{-2 n}\left(\eta_{n+1}-\eta_{n}\right) \text {. }
\end{aligned}
$$

Thus (if we put $l=0$ outside $E_{\delta}$ )

$$
\begin{aligned}
\left(\eta_{n+1}-\eta_{n}\right) \int_{2^{-N}}^{1-2-N} l^{2} d x \leqq & \sum_{n=1}^{2 n-2}\left(\eta_{n+1}-\eta_{n}\right) \int_{I_{h}} l^{2} d x \\
\leqq & 2^{-n+1} \int_{\eta_{n}}^{\eta_{n+1}} \int_{0}^{1}\left[\left(\frac{\partial u}{\partial x}\right)^{2}+\left(\frac{\partial u}{\partial y}\right)^{2}\right] d x d y \\
& +\epsilon\left(\eta_{n+1}-\eta_{n}\right) .
\end{aligned}
$$

Therefore

$$
\left(\eta_{n+1}-\eta_{n}\right) \int_{2^{-N}}^{1-2-N} l^{2} d x \leqq 2^{-n+1} D(u)+\epsilon\left(\eta_{n+1}-\eta_{n}\right) .
$$

Summing for $n \geqq N$ gives

$$
\left(1-\eta_{N}\right) \int_{2^{-N}}^{1-2-N} l^{2} d x \leqq 2^{-N+2} D(u)+\epsilon\left(1-\eta_{N}\right) .
$$

If we let $\eta_{n}$ converge to one so slowly that

$$
\sup 2^{n}\left(1-\eta_{n}\right)=\infty \text {, }
$$

then

$$
\int_{0}^{1} l^{2} d x<\epsilon
$$

and since the left-hand side is independent of $n$,

$$
\int_{0}^{1} l^{2} d x=0
$$

hence $l=0$ almost everywhere in $E_{\boldsymbol{\gamma}}$. Since $\delta$ is arbitrary we must have

$$
\lim _{y \rightarrow 1} u(x, y)=0
$$

for almost all $x$ in $[0,1]$. Similarly

$$
\lim _{y \rightarrow-1} u(x, y)=0
$$

for almost all $x$ in $[0,1]$. 
For almost all $x$ we then have

$$
u(x, y)=\int_{-1}^{v} \frac{\partial u}{\partial y} d y
$$

and by the Schwarz inequality

$$
u(x, y)^{2} \leqq 2 \int_{-1}^{1}\left[\left(\frac{\partial u}{\partial x}\right)^{2}+\left(\frac{\partial u}{\partial y}\right)^{2}\right] d y=\mu(x)
$$

But $\mu$ is summable since

$$
\int_{0}^{1} \mu(x) d x=2 D(u)
$$

Hence

$$
m(y)=\int_{0}^{1} u^{2} d x
$$

is continuous and $m(1)=m(-1)=0$. Moreover

$$
\begin{aligned}
m^{\prime}\left(y_{2}\right)-m^{\prime}\left(y_{1}\right) & =\left.2 \int_{0}^{1} u \frac{\partial u}{\partial y} d x\right|_{y=y_{2}}-\left.2 \int_{0}^{1} u \frac{\partial u}{\partial y} d x\right|_{y=y_{1}} \\
& =2 \int_{y_{1}}^{y_{2}} \int_{0}^{1}\left[\left(\frac{\partial u}{\partial x}\right)^{2}+\left(\frac{\partial u}{\partial y}\right)^{2}\right] d x d y,
\end{aligned}
$$

since $u$ is a harmonic function which vanishes for $x=0$ and $x=1$. Thus $m^{\prime}(y)$ is increasing whence $m(y)$ is convex. But $m(y)$ must vanish identically in the interval $-1 \leqq y \leqq 1$, since it is non-negative, convex, and vanishes at the end points. Hence $u \equiv 0$.

The function $u=y$ is single-valued on $V^{\prime}$ and has a finite Dirichlet integral, while $\partial u / \partial n=0$ on $R$. Thus we have the following result.

On the bounded Riemann surface $V^{\prime}$ the class $H O$ is empty, while there is a nonconstant harmonic function with a finite Dirichlet integral whose normal derivative vanishes on the relative boundary.

3. We form a Riemann surface $W^{\prime}$ in the strip $|y| \leqq 1$ by putting in replicas of $V^{\prime}$ in each rectangle $n \leqq x \leqq n+1$. Let $u$ be a bounded harmonic function with a finite Dirichlet integral defined on $W^{\prime}$. Then a slight modification in the argument of the preceding section shows that

$$
\lim _{y \rightarrow 1} u(x, y)=c
$$

for almost all $x$, and 


$$
\lim _{y \rightarrow-1} u(x, y)=c^{\prime}
$$

for almost all $x$.

Without loss of generality we may take $c^{\prime}=0$. Then

$$
u(x, y)=\int_{-1}^{y} \frac{\partial u}{\partial y} d y
$$

and by the Schwarz inequality

$$
u(x, y)^{2} \leqq 2 \int_{-1}^{y}\left(\frac{\partial u}{\partial y}\right)^{2} d y \leqq 2 \int_{-1}^{1}\left(\frac{\partial u}{\partial y}\right)^{2} d y=\mu(x)
$$

Since

$$
\int_{-\infty}^{\infty} \mu(x) \leqq D(u)<\infty,
$$

$\mu$ is summable and so

$$
m(y)=\int_{-\infty}^{\infty} u(x, y)^{2} d x
$$

exists and is continuous for $-1 \leqq y \leqq 1$. Also we have $c^{2} \leqq \mu(x)$, whence $c$ must be zero in order for $\mu$ to be summable on $(-\infty, \infty)$. Hence, $m\left(y_{1}\right)=m\left(y_{2}\right)=0$. By the Schwarz inequality we have

$$
\begin{aligned}
\left(\iint\left|u \frac{\partial u}{\partial y}\right| d x d y\right)^{2} & \leqq \iint u^{2} d x d y \iint\left(\frac{\partial u}{\partial y}\right)^{2} d x d y \\
& \leqq D(u)^{2}<\infty
\end{aligned}
$$

Thus by the Fubini theorem

$$
m_{1}(y)=\frac{1}{2} \int_{-\infty}^{\infty} u \frac{\partial u}{\partial y} d x
$$

exists for almost all $y$ and

$$
\begin{aligned}
m\left(y_{2}\right)-m\left(y_{1}\right) & =\frac{1}{2} \int_{-\infty}^{\infty} \int_{y_{1}}^{y_{2}} u \frac{\partial u}{\partial y} d y d x \\
& =\frac{1}{2} \int_{y_{1}}^{y_{2}} \int_{-\infty}^{\infty} u \frac{\partial u}{\partial y} d x d y \\
& =\int_{y_{1}}^{y_{2}} m_{1}(y) d y
\end{aligned}
$$


whence $m$ is absolutely continuous and

$$
m^{\prime}=m_{1} \text { a.e. }
$$

Let $y_{1}$ and $y_{2}$ be two values of $y$ for which $m_{1}$ exists, and take $N$ so large that $y_{1}<\eta_{N}, y_{2}<\eta_{N}$. Since

$$
\iint\left(\frac{\partial u}{\partial x}\right)^{2}+\left(\frac{\partial u}{\partial y}\right)^{2} d x d y<\infty
$$

we can find an arbitrarily large $x_{0}$ such that the fractional part of $x_{0}$ lies between zero and $2^{-N}$ and such that

$$
\int\left[\left(\frac{\partial u}{\partial x}\right)^{2}+\left(\frac{\partial u}{\partial y}\right)^{2}\right] d y<\frac{\epsilon^{2}}{2}
$$

on the two segments $x= \pm x_{0}$. On these segments

$$
\left(\int\left|\frac{\partial u}{\partial x}\right| d y\right)^{2} \leqq 2 \int\left(\frac{\partial u}{\partial x}\right)^{2} d y<\epsilon^{2}
$$

and so

$$
\int\left|\frac{\partial u}{\partial x}\right| d y<\epsilon
$$

We also take $x_{0}$ so large that

$$
\iint_{|x|>x_{0}}\left[\left(\frac{\partial u}{\partial x}\right)^{2}+\left(\frac{\partial u}{\partial y}\right)^{2}\right] d x d y<\epsilon
$$

and

$$
\int_{|x|>x_{0}}\left|u \frac{\partial u}{\partial y}\right| d x<\quad \text { for } y=y_{1}, y_{2} \text {. }
$$

Since the fractional part of $x_{0}$ lies between zero and $2^{-N}$, the region $\Omega$ on $W^{\prime}$ for which $|x|<x_{0}$ and $y_{1} \leqq y \leqq y_{2}$ has as its boundary the segments $|x|<x_{0}, y=y_{1}, y_{2}$ and the segments $y_{1}<y<y_{2},|x|=x_{0}$. Thus by Green's theorem

$$
\begin{aligned}
D_{\Omega}(u)=\int u \frac{\partial u}{\partial n}= & \int_{x=x_{0}} u \frac{\partial u}{\partial x} d y-\int_{x=-x_{0}} u \frac{\partial u}{\partial x} d y \\
& +\int_{y=y_{2}} u \frac{\partial u}{\partial x} d y-\int_{y=y_{1}} u \frac{\partial u}{\partial x} d y
\end{aligned}
$$

where the ranges of integration are $y_{1} \leqq y \leqq y_{2},-x_{0} \leqq x \leqq x_{0}$. 
Thus

$$
\left|D_{\Omega}(u)-m_{1}\left(y_{2}\right)+m_{1}\left(y_{2}\right)\right|<2 M \epsilon+2 \epsilon
$$

where $M$ is a bound for $|u|$. Consequently

$$
\left|\int_{y_{1}}^{y_{2}} \int_{-\infty}^{\infty}\left[\left(\frac{\partial u}{\partial x}\right)^{2}+\left(\frac{\partial u}{\partial y}\right)^{2}\right] d x d y-m_{1}\left(y_{2}\right)+m_{1}\left(y_{1}\right)\right|<(2 M+3) \epsilon .
$$

The left-hand side must be zero since it is independent of $\epsilon$, and so

$$
m_{1}\left(y_{2}\right)-m_{1}\left(y_{1}\right)=\int_{y_{1}}^{y_{2}} \int_{-\infty}^{\infty}\left[\left(\frac{\partial u}{\partial x}\right)^{2}+\left(\frac{\partial u}{\partial y}\right)^{2}\right] d x d y \geqq 0 .
$$

From this and the fact that

$$
m(y)=\int^{y} m_{1}(y) d y
$$

we conclude that $m(y)$ is convex. As a result $m(y) \equiv 0$ in the interval $-1 \leqq y \leqq 1$, since it is a non-negative convex function vanishing at the ends of the interval. This implies $u \equiv 0$, and because of the identity $O_{H D}=O_{H B}$ we have the following proposition:

The Riemann surface $W^{\prime}$ has no nonconstant harmonic functions on it with a finite Dirichlet integral, while the function $u=y$ is a harmonic function which is defined, single-valued, and bounded on $W^{\prime}$.

\section{BIBLIOGRAPHY}

1. Lars V. Ahlfors and H. L. Royden, A counterexample in the classification of open Riemann surfaces, Annales Academiae Scientiarum Fennicae. Ser. AI.

2. H. L. Royden, Harmonic functions on open Riemann surfaces, Trans. Amer. Math. Soc. vol. 73 (1952) pp. 40-94.

STANFORD UNIVERSITY 